\title{
Phytoconstituents with cytotoxic activity from Ulmus pumila L.
}

\author{
Farouk R. Melek ${ }^{1 *}$, Soheir M. El Zalabani ${ }^{2}$, Neveen S. Ghaly ${ }^{1}$, Omar M. Sabry², Walid Fayad ${ }^{3}$, Ann G. Boulis ${ }^{1}$ \\ ${ }^{1}$ Chemistry of Natural Compounds Department, National Research Centre, 60014618, Dokki, Giza, 12622, Egypt \\ ${ }^{2}$ Pharmacognosy Department, Faculty of Pharmacy, Cairo University, Cairo, 11562, Egypt \\ ${ }^{3}$ Drug Bioassay-Cell Culture Laboratory, Pharmacognosy Department, National Research Centre, Dokki, Giza, 12622, Egypt
}

\begin{tabular}{l}
\hline ARTICLE INFO \\
\hline Received on: $22 / 12 / 2020$ \\
Accepted on: $02 / 02 / 2021$ \\
Available online: $05 / 05 / 2021$
\end{tabular}

\section{Key words:}

Ulmus pumila L., triterpenoids, phenolics, cytotoxic activity.

\begin{abstract}
The phytochemical examination of the stem bark and leafy branches of Ulmus pumila L. gave rise to the separation of 13 compounds, recognized as Friedelin, $3 \beta$-acetoxyurs-11-en-13 $\beta$, 28-olide, $3 \beta$-O-acetyl ursolic acid, $3 \beta$-O-acetyl oleanolic acid, $\beta$-sitosterol, stigmasterol, betulinic acid, methyl ursolate, methyl oleanolate, kaempferol-3- $O$-rutinoside, quercetin-3- $O-\beta$-D-glucopyranoside, quercetin-3- $O-\beta$-D-galactopyranoside, and caffeic acid. Their structures were elucidated using chemical and spectroscopic methods (ultraviolet, Infrared, EI-MS, ${ }^{1} \mathrm{H}-\mathrm{NMR}$, and ${ }^{13} \mathrm{C}-\mathrm{NMR}$ ) and by comparison with literature data. The cytotoxic potential of the crude methanol extract of the stem bark, besides the isolated triterpenoids, was tested against five human carcinoma cell lines, namely human colorectal carcinoma (HCT-116), human breast adenocarcinoma (MCF-7), human hepatocellular carcinoma (HepG2), human osteosarcoma (HOS), and human pulmonary adenocarcinoma (A549) cell lines. Betulinic acid exhibited a cytotoxic potential against MCF-7, HCT-116, and A549 cell lines with half maximal inhibitory concentration $\left(\mathrm{IC}_{50}\right)$ values equal to $22.39 \pm 0.09$ $\mu \mathrm{M}, 22.29 \pm 0.05 \mu \mathrm{M}$, and $42.33 \pm 0.06 \mu \mathrm{M}$, respectively. Meanwhile, the remaining triterpenoids showed a cytotoxic potential against HCT-116 and MCF-7 cell lines, with $\mathrm{IC}_{50}$ values ranging from $48.91 \pm 0.12$ to $78.98 \pm 0.07 \mu \mathrm{M}$. The demonstrated cytotoxic potential of betulinic acid suggests its use as a lead compound for anticancer therapy.
\end{abstract}

\section{INTRODUCTION}

The family Ulmaceae, commonly known as the elm family, comprises about 6 genera and 45 species (Encyclopaedia Britannica, 2019). Ulmaceae members are evergreen or deciduous trees and shrubs distributed throughout the north temperate zone. Ulmus species, about 35 in number, are primarily distributed in Asia, Europe, and North America (Richens, 1983; Watson and Dallwitz, 1992). Previous studies on genus Ulmus reported the presence of various types of phytoconstituents like terpenoids (Martín-Benito et al., 2011), steroids (Martín-Benito et al., 2011), phenolics (Zhou et al., 2017), and polysaccharides (Lee et al., 2018). From a bioactivity standpoint, Ulmus species were reported to exhibit antibiotic (You et al., 2013), antifungal (Burden and Kemp, 1984), antioxidant (Bora et al., 2017; Joo et al., 2014; Mina et al., 2016), anti-inflammatory (Joo et al., 2014; Mina

${ }^{*}$ Corresponding Author

Farouk R. Melek, National Research Centre, 33El Behouth st., Dokki, Giza, Egypt. E-mail: frmelek1@gmail.com et al., 2016), hepatoprotective (Boudaoud-Ouahmed et al., 2015), neuroprotective (So et al., 2019), antiangiogenic (Jung et al., 2007), cytotoxic (Wang et al., 2004; Wang et al., 2006), anticancer (Hamed et al., 2015), and antiviral (Hamed et al., 2015) effects.

Ulmus pumila L., renowned as Asiatic elm, Chinese elm, and dwarf elm, is a deciduous tree belonging to central Asia. In folk medicine, its leaf and stem bark extracts are employed as diuretic, demulcent, antipyretic, and laxative remedies (Duke and Ayensu, 1985). Ulmus pumila L. was reported to possess large amounts of phenols and flavonoids with potent antioxidant activities (Kim et al., 2010). In addition, previous studies on the constituents of the root bark of this species led to the characterization of two potentially cytotoxic sesquiterpenoids, namely, mansonones $\mathrm{E}$ and F (Wang et al., 2004), as well as various bioactive triterpenoids (Wang et al., 2006). Moreover, four triterpenoids, namely, oleanolic acid, friedelin, maslinic acid, and arjunolic acid, were also isolated from the methanol extract of $U$. pumila L. (Ghosh et al., 2012). Furthermore, a recent phytochemical study on the stem bark extract of $U$. pumila L. led to separation of Icariside $\mathrm{E}_{4}$ which strongly prohibited nitric oxide generation in LPSactivated macrophages (Joo et al., 2014). As an extension to our 
interest in exploring bioactive compounds from natural sources, we described in this report the isolation and identification of 13 compounds from $U$. pumila L. stem bark and leafy branches. The cytotoxic activity of the stem bark methanol extract and some of the isolated triterpenoids against five human carcinoma cell lines was also reported.

\section{MATERIALS AND METHODS}

\section{Plant material}

The stem bark and leafy branches of $U$. pumila L. were gathered from Orman Botanical Garden, Giza, Egypt, in January and February 2018. A voucher specimen, encoded M131, was submitted to the National Research Centre herbarium, Giza, Egypt.

\section{General experimental procedures}

Vacuum liquid chromatography (VLC) was achieved with silica gel H 60 (E-Merck, Darmstadt, Germany) and polyamide 11 (E-Merck, Darmstadt, Germany). Preparative and analytical thin layer chromatography were carried out using silica gel (E-Merck, Darmstadt, Germany). Chromatograms were first visualized under ultraviolet (UV) light and then sprayed with $20 \%$ sulfuric acid in methanol or ferric chloride reagent. Column chromatography was performed using Sephadex LH-20 (SigmaAldrich, St. Louis, MO). Infrared (IR) spectra were run on a JASCO FT/IR-6100 Fourier Transform IR Spectrometer (Oklahoma, USA). Mass spectra (MS) were acquired by means of a Thermo ISQ Single Quadrupole Mass Spectrometer (THERMO Scientific Corporation, USA). UV spectra were displayed on a Shimadzu Double Beam Spectrophotometer UV-1650 (Shimadzu, Japan). Nuclear magnetic resonance (NMR) spectra were obtained via a Bruker High Performance Digital FT-NMR-Spectrophotometer Avance III HD ( ${ }^{1} \mathrm{H}-\mathrm{NMR}$ : $400 \mathrm{MHz},{ }^{13} \mathrm{C}-\mathrm{NMR}$ : $100 \mathrm{MHz}$, Bremen, Germany). Chemical shifts were expressed on the $\delta$ scale and tetramethylsilane was used as an internal standard.

\section{Extraction and isolation of stem bark and leafy branch constituents}

Air-dried powdered stem bark and leafy branches (1 and $1.2 \mathrm{~kg}$, resp.) were separately extracted with methanol (5 1 $\times 3$ ) at room temperature. Upon vacuum evaporation, the stem bark extract yielded a reddish-brown residue $(50 \mathrm{~g})$ and the leafy branch extract yielded a dark green residue $(110 \mathrm{~g})$. A portion of each dried extract (45 g of stem bark extract and $100 \mathrm{~g}$ of leafy branch extract) was individually suspended in distilled water (500 $\mathrm{ml})$ and then partitioned with dichloromethane $(250 \mathrm{ml} \times 5)$, ethyl acetate $(250 \mathrm{ml} \times 5)$, and water-saturated $n$-butanol $(300 \mathrm{ml} \times 4)$, in succession. The solvent-free dichloromethane fraction $(19 \mathrm{~g})$ from the stem bark extract and ethyl acetate fraction from leafy branch extract (4.2 g) were subjected to VLC (silica gel $500 \mathrm{~g}$, and polyamide $11250 \mathrm{~g}$, resp.).

Elution of the silica gel bed was started using $n$-hexane and continued with $n$-hexane with $5 \%$ increments of acetone up to $50 \%$. Thirty fractions, $100 \mathrm{ml}$ each, were collected and examined by TLC (solvent system, $n$-hexane- $\mathrm{CH}_{2} \mathrm{Cl}_{2}-\mathrm{MeOH}, \quad 10: 10: 1$ $v / v / v$; spraying reagent, $20 \%$ sulfuric acid, followed by heating at $110^{\circ} \mathrm{C}$ ). Fractions eluted with $10 \%$ acetone, with compound 1 as the major component, were combined and rechromatographed on a Sephadex LH-20 column (eluent, $\mathrm{CH}_{2} \mathrm{Cl}_{2}-\mathrm{MeOH} 3: 2 v / v$ ) to yield pure compound 1 ( $80 \mathrm{mg}$ ). The $25 \%$ acetone fractions (similar TLC pattern, three major spots) were pooled and the solvent was evaporated. The residue was subjected to repeated PTLC (solvent system, $n$-hexane- $\mathrm{CH}_{2} \mathrm{Cl}_{2}-\mathrm{MeOH}$ 10:10:1 $v / v / v$, triple development), followed by repeated chromatography on Sephadex LH-20 columns (eluent, $\mathrm{CH}_{2} \mathrm{Cl}_{2}-\mathrm{MeOH}$ 3:2 v/v) to yield compound 2 (24 mg), compound 3 (26 mg) slightly contaminated with compound 4, and a mixture of compounds 5 and $\mathbf{6}$ (12.5 $\mathrm{mg}$ ). The $35 \%$ acetone fractions (similar TLC pattern, two major spots) were pooled, evaporated, and subjected to repeated PTLC (solvent system, $n$-hexane- $\mathrm{CH}_{2} \mathrm{Cl}_{2}-\mathrm{MeOH}, 10: 10: 1 \mathrm{v} / \mathrm{v} / \mathrm{v}$, triple development), followed by repeated purification on Sephadex LH20 columns (eluent, $\mathrm{CH}_{2} \mathrm{Cl}_{2}-\mathrm{MeOH} 3: 2 v / v$ ) to yield compound 7 $(4.5 \mathrm{mg})$ and a mixture of compounds 8 and 9 (11.5 mg).

Elution of the polyamide 11 bed was started with $\mathrm{H}_{2} \mathrm{O}$ and then by $10 \%$ increments of $\mathrm{MeOH}$ up to $80 \%$. Eighty fractions, $100 \mathrm{ml}$ each, were collected and monitored by TLC (solvent system, EtOAc-MeOH-H $\mathrm{O}_{2}$ 30:5:4 v/v/v). Spots were detected in visible and UV (365 nm) lights, before and after exposure to ammonia vapor or spraying with ferric chloride. Fractions eluted with 10,20, and $30 \% \mathrm{MeOH}$, with compound $\mathbf{1 0}$ as the major component, were mixed. After evaporating the solvent, the residue was subjected to column chromatography (Sephadex LH-20; eluent, $\left.\mathrm{H}_{2} \mathrm{O}-\mathrm{MeOH} 1: 1 v / v\right)$ to yield compound $10(11.5$ $\mathrm{mg}$ ). Fractions eluted with $50 \%$ and $60 \% \mathrm{MeOH}$ were combined based on TLC analysis. After solvent evaporation, the residue was chromatographed on a Sephadex LH-20 column (eluent, $\mathrm{H}_{2} \mathrm{O}-$ $\mathrm{MeOH} 1: 1 v / v)$ to yield a mixture of compounds $\mathbf{1 1}$ and 12 (41.1 $\mathrm{mg}$ ) together with compound $\mathbf{1 3}(10.5 \mathrm{mg})$.

\section{Identification of the isolated compounds}

Compounds 1-13 shown in Figure 1 were identified based on the following spectral data:

Friedelin (compound 1): EI-MS $(\mathrm{m} / \mathrm{z}$, relative abundance); $426\left([\mathrm{M}]^{+}, \mathrm{C}_{30} \mathrm{H}_{50} \mathrm{O}, 2 \%\right), 411\left([\mathrm{M}-\mathrm{Me}]^{+}, 1 \%\right), 341$ (1\%), 273 (18\%), 205 (28\%), 123 (52\%), 55 (100\%). IR (KBr, $\left.\mathbf{c m}^{-1}\right) ; 2,930,2,868\left(v_{\mathrm{C}-\mathrm{H}}\right), 1,710\left(v_{\mathrm{C}=\mathrm{O}}\right), 1,453,1,385\left(v_{\mathrm{C}-\mathrm{H}}\right)$. ${ }^{1} \mathbf{H}-\mathbf{N M R}\left(\mathbf{C D C l}_{3}, \mathbf{p p m}\right) ; 0.73(3 \mathrm{H}, s, \mathrm{Me}-24), 0.88(3 \mathrm{H}, s, \mathrm{Me}-$ 25), $0.89(3 \mathrm{H}, d, J=6.0 \mathrm{~Hz}, \mathrm{Me}-23), 0.96$ (3H, $s, \mathrm{Me}-29), 1.02$ (6H, $s, \mathrm{Me}-26, \mathrm{Me}-30), 1.06$ (3H, $s, \mathrm{Me}-27), 1.19$ (3H, $s, \mathrm{Me}-28)$, 1.69 (1H, $m, \mathrm{H}-1 \mathrm{a}), 1.97$ (1H, $m, \mathrm{H}-1 \mathrm{~b}), 2.26(1 \mathrm{H}, q, J=6.4 \mathrm{~Hz}$, H-4), 2.32 (1H, $m, \mathrm{H}-2 \mathrm{a}), 2.40$ (1H, $m, \mathrm{H}-2 \mathrm{~b}) .{ }^{13} \mathbf{C}-\mathbf{N M R}\left(\mathbf{C D C l}_{3}\right.$, ppm); 22.3 (C-1), 41.5 (C-2), 213.2 (C-3), 58.2 (C-4), 42.1 (C-5), 41.3 (C-6), 18.2 (C-7), 53.1 (C-8), 37.4 (C-9), 59.5 (C-10), 35.6 (C-11), 30.5 (C-12), 39.7 (C-13), 38.3 (C-14), 32.4 (C-15), 36.0 (C-16), 30.0 (C-17), 42.8 (C-18), 35.3 (C-19), 28.2 (C-20), 32.8 (C-21), 39.3 (C-22), 6.8 (C-23), 14.7 (C-24), 18.0 (C-25), 20.3 (C26), 18.7 (C-27), 32.1 (C-28), 35.0 (C-29), 31.8 (C-30).

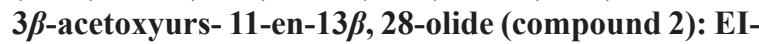
MS ( $m / z$, relative abundance); $496\left([\mathrm{M}]^{+}, \mathrm{C}_{32} \mathrm{H}_{48} \mathrm{O}_{4}, 0.2 \%\right), 452$ $\left(\left[\mathrm{M}-\mathrm{CO}_{2}\right]^{+}, 0.1 \%\right), 436\left(\left[\mathrm{M}-\mathrm{CH}_{3} \mathrm{COOH}\right]^{+}, 0.5 \%\right), 249(2 \%), 248$ (3\%), 203 (6\%), 165 (47\%), 135 (37\%), 123 (45\%), 109 (68\%), 81 (60\%), 69 (100\%). IR (KBr, cm $\left.{ }^{-1}\right) ; 2,925,2,856\left(v_{\mathrm{C}-\mathrm{H}}\right), 1,756$ (sh.) $\left(v_{\mathrm{C}=0}, \gamma\right.$-lactone $), 1,732\left(v_{\mathrm{C}=0}\right.$, ester $), 1,645\left(v_{\mathrm{C}=\mathrm{C}}\right), 1,460$, $1,378\left(v_{\mathrm{C}-\mathrm{H}}\right), 1,243\left(v_{\mathrm{C}-\mathrm{O}}\right.$, acetate $), 1,023\left(v_{\mathrm{C}-\mathrm{O}}\right) .{ }^{\mathbf{1}} \mathbf{H}-\mathbf{N M R}\left(\mathbf{C D C l}_{3}\right.$, ppm); 0.87 (6H, $s, \mathrm{Me}-23, \mathrm{Me}-25), 0.93(3 \mathrm{H}, d, J=5.5 \mathrm{~Hz}, \mathrm{Me}-$ 29), 0.98 (3H, $s, \mathrm{Me}-27), 1.09(3 \mathrm{H}, d, J=4.0 \mathrm{~Hz}, \mathrm{Me}-30), 1.18$ 


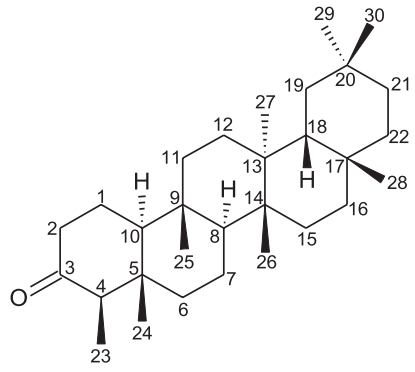

1

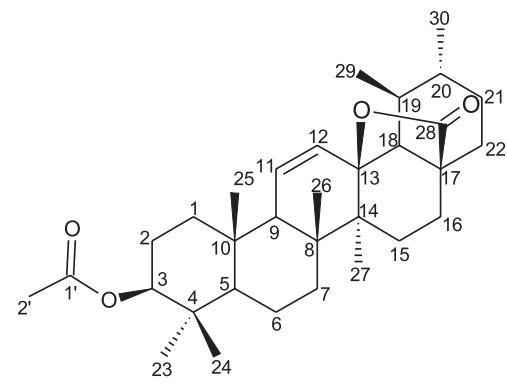

2
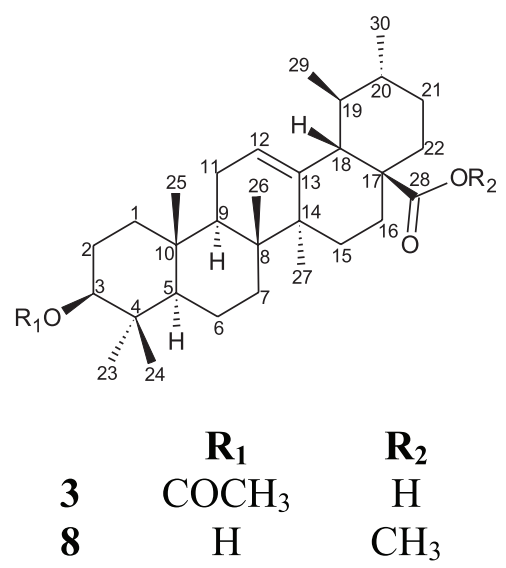

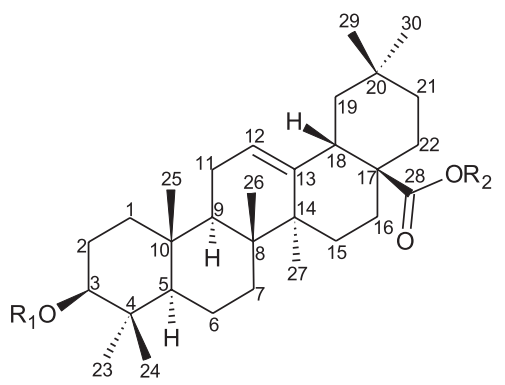

$\mathbf{R}_{1}$

$4 \mathrm{COCH}_{3}$

$9 \quad \mathrm{H} \quad \mathrm{CH}_{3}$

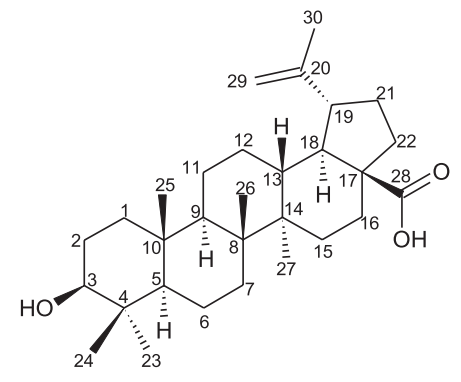

7<smiles>[R]c1cc(-c2oc3cc(O)cc(O)c3c(=O)c2OCCO)ccc1O</smiles>

$\mathbf{R}_{1}$

$10 \alpha$-L-Rhamnopyranosyl-(1-6)- $\beta$-Dglucopyranosyl

11

12

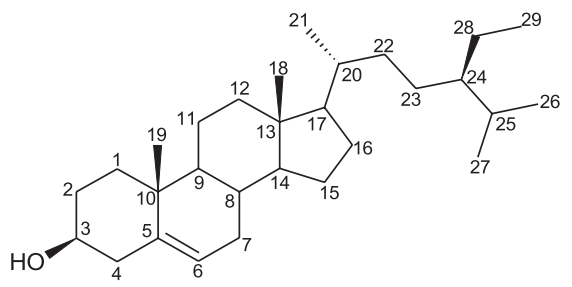

5

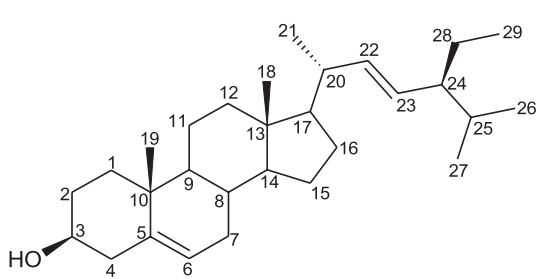

6<smiles>O=C(O)/C=C/c1ccc(O)c(O)c1</smiles>

13
$\mathbf{R}_{2}$

$\mathrm{H}$

$\mathrm{OH}$

$\mathrm{OH}$

Figure 1. Phytoconstituents identified in U. pumila L. grown in Egypt.

$(6 \mathrm{H}, s, \mathrm{Me}-24, \mathrm{Me}-26), 1.99(3 \mathrm{H}, s$, Acetate Me), $4.43(1 \mathrm{H}, d d, J$ $=10.3,5.8 \mathrm{~Hz}, \mathrm{H}-3), 5.47(1 \mathrm{H}, d d, J=10.3,2.7 \mathrm{~Hz}, \mathrm{H}-11), 5.88$ $(1 \mathrm{H}, d, J=10.3 \mathrm{~Hz}, \mathrm{H}-12),{ }^{13} \mathbf{C}-\mathbf{N M R}\left(\mathbf{C D C l}_{3}, \mathbf{p p m}\right) ; 38.1(\mathrm{C}-1)$, 23.3 (C-2), 80.6 (C-3), 37.8 (C-4), 54.8 (C-5), 18.0 (C-6), 31.2 (C-7), 41.9 (C-8), 52.9 (C-9), 36.3 (C-10), 128.9 (C-11), 133.3 (C-12), 89.6 (C-13), 41.7 (C-14), 25.5 (C-15), 22.8 (C-16), 45.1
(C-17), 60.6 (C-18), 38.0 (C-19), 40.3 (C-20), 30.8 (C-21), 31.3 (C-22), 27.7 (C-23), 16.1 (C-24), 16.1 (C-25), 18.9 (C-26), 17.9 (C-27), 179.9 (C-28), 17.6 (C-29), 19.2 (C-30), 171.0 (Acetate C $=\mathrm{O}), 21.3$ (Acetate Me).

3及-O-acetyl ursolic acid (compound 3): EI-MS ( $m / z$, relative abundance); $498\left([\mathrm{M}]^{+}, \mathrm{C}_{32} \mathrm{H}_{50} \mathrm{O}_{4}, 17 \%\right), 454([\mathrm{M}-$ 
$\mathrm{CO} 2]+, 6 \%), 438([\mathrm{M}-\mathrm{CH} 3 \mathrm{COOH}]+, 1 \%), 249(69 \%), 248(58 \%)$, 235 (13\%), 203 (98\%), 202 (19\%), 190 (26\%), 189 (65\%), 133 (100\%), 123 (34\%), 120 (94\%), 109 (24\%). IR (KBr, cm $\left.{ }^{-1}\right)$; 3, 431 $\left(v_{\mathrm{O}-\mathrm{H}}\right), 2,925,2,854\left(v_{\mathrm{C}-\mathrm{H}}\right), 1,728\left(v_{\mathrm{C}=\mathrm{O}}\right), 1,630\left(v_{\mathrm{C}=\mathrm{C}}\right), 1,440,1,383$ $\left(v_{\mathrm{C}-\mathrm{H}}\right), 1,253\left(v_{\mathrm{C}-\mathrm{O}}\right.$, acetate $), 1,025\left(v_{\mathrm{C}-\mathrm{O}}\right) .{ }^{1} \mathbf{H}-\mathbf{N M R}\left(\mathbf{C D C l}_{3}, \mathbf{p p m}\right)$; $0.78(3 \mathrm{H}, s, \mathrm{Me}-27), 0.87$ (3H, $s, \mathrm{Me}-26), 0.89$ (3H, $s, \mathrm{Me}-25)$, $0.97(6 \mathrm{H}, d, J=6.6 \mathrm{~Hz}, \mathrm{Me}-29, \mathrm{Me}-30), 1.09(3 \mathrm{H}, s, \mathrm{Me}-24), 1.27$ $(3 \mathrm{H}, s, \mathrm{Me}-23), 2.07(3 \mathrm{H}, s$, Acetate Me), $2.20(1 \mathrm{H}, d, J=11.2$ $\mathrm{Hz}, \mathrm{H}-18), 4.52$ (1H, $m, \mathrm{H}-3), 5.25$ (1H, br $s, \mathrm{H}-12) .{ }^{13} \mathbf{C}-\mathbf{N M R}$ (CDCl $\left.{ }_{3}, \mathbf{p p m}\right) ; 38.3$ (C-1), 23.6 (C-2), 81.0 (C-3), 37.7 (C-4), 55.3 (C-5), 18.2 (C-6), 32.8 (C-7), 39.5 (C-8), 47.5 (C-9), 36.9 (C-10), 23.3 (C-11), 125.7 (C-12), 138.0 (C-13), 41.9 (C-14), 28.0 (C-15), 24.0 (C-16), 48.0 (C-17), 52.5 (C-18), 38.8 (C-19), 39.0 (C-20), 30.6 (C-21), 36.7 (C-22), 28.1 (C-23), 17.1 (C-24), 15.5 (C-25), 16.7 (C-26), 23.6 (C-27), 184.0 (C-28), 17.0 (C-29), 21.2 (C-30), 171.1 (Acetate $\mathrm{C}=\mathrm{O}$ ), 21.3 (Acetate $\mathrm{Me}$ ).

3 $\beta$-O -acetyl oleanolic acid (compound 4): EI-MS ( $m / z$, relative abundance); $498\left([\mathrm{M}]^{+}, \mathrm{C}_{32} \mathrm{H}_{50} \mathrm{O}_{4}, 17 \%\right), 454([\mathrm{M}-$ $\mathrm{CO} 2]+, 6 \%), 438([\mathrm{M}-\mathrm{CH} 3 \mathrm{COOH}]+, 1 \%), 249(69 \%), 248(58 \%)$, 235 (13\%), 203 (98\%), 202 (19\%), 190 (26\%), 189 (65\%), 133 (100\%), 123 (34\%), 120 (94\%), 109 (24\%). IR (KBr, $\left.\mathbf{c m}^{-1}\right) ; 3,431$ $\left(v_{\mathrm{O}-\mathrm{H}}\right), 2,925,2,854\left(v_{\mathrm{C}-\mathrm{H}}\right), 1,728\left(v_{\mathrm{C}=\mathrm{O}}\right), 1,630\left(v_{\mathrm{C}=\mathrm{C}}\right), 1,440,1,383$ $\left(v_{\mathrm{C}-\mathrm{H}}\right), 1,253\left(v_{\mathrm{C}-\mathrm{O}}\right.$, acetate $), 1,025\left(v_{\mathrm{C}-\mathrm{O}}\right) .{ }^{1} \mathbf{H}-\mathbf{N M R}\left(\mathbf{C D C l}_{3}, \mathbf{p p m}\right)$; $0.76(3 \mathrm{H}, s, \mathrm{Me}-26), 0.84$ (6H, $s, \mathrm{Me}-23, \mathrm{Me}-24), 0.92(3 \mathrm{H}, s, \mathrm{Me}-$ 30), 0.95 (6H, s, Me-25, Me 29), 1.14 (3H, s, Me-27), 2.07 (3H, $s$, Acetate Me), 4.52 (1H, $m, \mathrm{H}-3), 5.29(1 \mathrm{H}, b r s, \mathrm{H}-12) .{ }^{13} \mathbf{C}$-NMR (CDCl, ppm); 38.1 (C-1), 23.6 (C-2), 81.0 (C-3), 37.7 (C-4), 55.3 (C-5), 18.2 (C-6), 32.6 (C-7), 39.3 (C-8), 47.5 (C-9), 36.9 (C-10), 22.8 (C-11), 122.5 (C-12), 143.6 (C-13), 41.6 (C-14), 27.7 (C-15), 23.4 (C-16), 46.6 (C-17), 40.9 (C-18), 45.8 (C-19), 30.7 (C-20), 33.8 (C-21), 32.5 (C-22), 28.1 (C-23), 16.7 (C-24), 15.4 (C-25), 17.2 (C-26), 25.9 (C-27), 184.3 (C-28), 33.1 (C-29), 23.6 (C-30), 171.1 (Acetate $\mathrm{C}=\mathrm{O}), 21.3($ Acetate $\mathrm{Me})$.

$\boldsymbol{\beta}$-Sitosterol (compound 5): EI-MS $(\mathrm{m} / \mathrm{z}$, relative abundance); $414\left([\mathbf{M}]+, \mathrm{C}_{29} \mathrm{H}_{50} \mathrm{O}, 85 \%\right), 399$ ([M-Me]+, 14\%); 396 ([M-H2O]+, 19\%), 381 (30\%), 273 (4\%), 255 (28\%), 213 (80\%), 161 (31\%), 133 (100\%), 105 (83\%). ${ }^{1} \mathbf{H}-\mathbf{N M R}\left(\mathbf{C D C l}_{3}\right.$, ppm); 0.70 (3H, s, Me-19), 0.83-0.89 (9H, $m$, Me-26, Me-27, Me29), $0.94(3 \mathrm{H}, d, J=6.6 \mathrm{~Hz}, \mathrm{Me}-21), 1.03(3 \mathrm{H}, s, \mathrm{Me}-18), 3.67$ $(1 \mathrm{H}, m, \mathrm{H}-3), 5.37(1 \mathrm{H}, d, J=4.6 \mathrm{~Hz}, \mathrm{H}-6)$.

Stigmasterol (compound 6): EI-MS ( $\mathrm{m} / \mathrm{z}$, relative abundance); $412\left([\mathrm{M}]^{+}, \mathrm{C}_{29} \mathrm{H}_{48} \mathrm{O}, 8 \%\right), 397$ ([M-Me] $\left.{ }^{+}, 28 \%\right), 394$ $\left(\left[\mathbf{M}-\mathrm{H}_{2} \mathbf{O}^{+}, 1 \%\right), 379(0.01 \%), 351(0.1 \%), 273(4 \%), 271(9 \%)\right.$, $257(2 \%), 255(28 \%), 229(36 \%), 213(80 \%), 133(100 \%), 107$ (79\%), 105 (83\%). ${ }^{1}$ H-NMR (CDCl ${ }_{3}$, ppm); 0.70 (3H, s, Me-19), $0.83-0.89$ (9H, $m, \mathrm{Me}-26, \mathrm{Me}-27, \mathrm{Me}-29), 0.94(3 \mathrm{H}, d, J=6.6 \mathrm{~Hz}$, Me-21), 1.31 (3H, $s, \mathrm{Me}-18), 3.55$ (1H, $m, \mathrm{H}-3), 5.04(1 \mathrm{H}, d d, J$ $=16.0,8.0 \mathrm{~Hz}, \mathrm{H}-22), 5.18(1 \mathrm{H}, d d, J=16.0,8.0 \mathrm{~Hz}, \mathrm{H}-23), 5.37$ $(1 \mathrm{H}, d, J=4.6 \mathrm{~Hz}, \mathrm{H}-6)$.

Betulinic acid (compound 7): EI-MS $(m / z$, relative abundance); $456\left(\left[\mathrm{M}^{+}, \mathrm{C}_{30} \mathrm{H}_{48} \mathrm{O}_{3}, 5 \%\right), 441\left([\mathrm{M}-\mathrm{Me}]^{+}, 6 \%\right)\right.$, $411\left([\mathrm{M}-\mathrm{COOH}]^{+}, 0.04 \%\right), 248(26 \%), 220(31 \%), 207(27 \%)$, 203 (43\%), 189 (100\%), 187 (31\%), 175 (18\%), 173 (27\%), 135 (82\%), 119 (94\%). IR (KBr, cm $\left.\mathbf{~ c m}^{-1}\right) ; 3,439\left(v_{\mathrm{O}-\mathrm{H}}\right), 2,926$ and 2,860 $\left(v_{\mathrm{C}-\mathrm{H}}\right), 1,678\left(\right.$ sh., $\left.v_{\mathrm{C}=\mathrm{O}}\right), 1,641\left(v_{\mathrm{C}=\mathrm{C}}\right), 1,456,1,381\left(v_{\mathrm{C}-\mathrm{H}}\right), 1,267$, $1,027\left(v_{\mathrm{C}-\mathrm{O}}\right) .{ }^{1} \mathbf{H}-\mathbf{N M R}\left(\mathbf{C D C l}_{\mathbf{3}}, \mathbf{p p m}\right) ; 0.68(3 \mathrm{H}, s, \mathrm{Me}-24), 0.75$ $(3 \mathrm{H}, s, \mathrm{Me}-25), 0.87$ (3H, $s, \mathrm{Me}-23), 0.90(3 \mathrm{H}, s, \mathrm{Me}-27), 0.91$
$(3 \mathrm{H}, s, \mathrm{Me}-26), 1.62(3 \mathrm{H}, s, \mathrm{Me}-30), 1.90(1 \mathrm{H}, m, \mathrm{H}-18), 2.93(1 \mathrm{H}$, $t d, J=10.6,4.7 \mathrm{~Hz}, \mathrm{H}-19), 3.12(1 \mathrm{H}, d d, J=11.1,4.8 \mathrm{~Hz}, \mathrm{H}-3)$, 4.54 (1H, br s, H-29a), 4.67 (1H, br s, H-29b).

Methyl ursolate (compound 8): EI-MS $(\mathrm{m} / \mathrm{z}$, relative abundance); $470\left(\left[\mathrm{M}^{+}, \mathrm{C}_{31} \mathrm{H}_{50} \mathrm{O}_{3}, 25 \%\right), 262(1 \%), 249(51 \%)\right.$, $208(0.1 \%), 203$ (18\%), $191(22 \%), 189(5 \%), 175(27 \%), 133$ (27\%), 123 (1\%), 120 (100\%), 109 (29\%). IR (KBr, cm $\left.{ }^{-1}\right) ; 3,427$ $\left(v_{\mathrm{O}-\mathrm{H}}\right), 2,925,2,856\left(v_{\mathrm{C}-\mathrm{H}}\right), 1,730\left(\mathrm{sh} ., v_{\mathrm{C}=\mathrm{O}}\right), 1,631\left(v_{\mathrm{C}=\mathrm{C}}\right), 1,458$, $1,381\left(v_{\mathrm{C}-\mathrm{H}}\right), 1,277,1,029\left(v_{\mathrm{C}-\mathrm{O}}\right) .{ }^{1} \mathbf{H}-\mathbf{N M R}\left(\mathbf{C D C l}_{3}, \mathbf{p p m}\right) ; 0.76$ $(3 \mathrm{H}, s, \mathrm{Me}-27), 0.86(3 \mathrm{H}, s, \mathrm{Me}-26), 0.88(3 \mathrm{H}, s, \mathrm{Me}-25), 0.93$ $(6 \mathrm{H}, d, J=6.8 \mathrm{~Hz}, \mathrm{Me}-29, \mathrm{Me}-30), 1.10(3 \mathrm{H}, s, \mathrm{Me}-24), 1.26(3 \mathrm{H}$, $s, \mathrm{Me}-23), 2.12(1 \mathrm{H}, d, J=11.3 \mathrm{~Hz}, \mathrm{H}-18), 3.15(1 \mathrm{H}, d d, J=10.3$, $4.3 \mathrm{~Hz}, \mathrm{H}-3), 3.60$ (3H, $s, \mathrm{MeOOC}-28)$ and 5.18 (1H, br s, H-12). ${ }^{13} \mathbf{C}-\mathbf{N M R}\left(\mathbf{C D C l}_{3}\right.$, ppm); 38.8 (C-1), 27.2 (C-2), 79.1 (C-3), 38.8 (C-4), 55.2 (C-5), 18.3 (C-6), 32.9 (C-7), 39.5 (C-8), 47.5 (C-9), 37.1 (C-10), 17.0 (C-11), 125.7 (C-12), 138.0 (C-13), 42.0 (C-14), 28.1 (C-15), 24.7 (C-16), 48.0 (C-17), 52.6 (C-18), 39.3 (C-19), 38.8 (C-20), 30.6 (C-21), 36.7 (C-22), 28.1 (C-23), 15.5 (C-24), 15.6 (C-25), 17.0 (C-26), 23.4 (C-27), 178.3 (C-28), 23.6 (C-29), 21.2 (C-30), 51.5 (MeOOC-28).

Methyl oleanolate (compound 9): EI-MS ( $m / z$, relative abundance); $470\left([\mathrm{M}]^{+}, \mathrm{C}_{31} \mathrm{H}_{50} \mathrm{O}_{3}, 25 \%\right), 262(1 \%), 249$ (51\%), 208 (0.1\%), 203 (18\%), 191 (22\%), 189 (5\%), 175 (27\%), 133 (27\%), 123 (1\%), 120 (100\%), 109 (29\%). IR (KBr, $\left.\mathbf{c m}^{-1}\right)$;

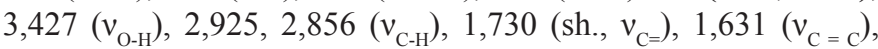
1,458, 1,381 ( $\left.v_{\mathrm{C}-\mathrm{H}}\right), 1,277,1,029\left(v_{\mathrm{C}-\mathrm{O}}\right) .{ }^{\mathbf{1}} \mathbf{H}-\mathbf{N M R}\left(\mathbf{C D C l}_{3}, \mathbf{p p m}\right)$; $0.71(3 \mathrm{H}, s, \mathrm{Me}-26), 0.80(3 \mathrm{H}, s, \mathrm{Me}-24), 0.87$ (3H, $s, \mathrm{Me}-23)$, $0.88(3 \mathrm{H}, s, \mathrm{Me}-30), 0.92(3 \mathrm{H}, s, \mathrm{Me}-29), 1.01$ (3H, $s, \mathrm{Me}-25)$, $1.07(3 \mathrm{H}, s, \mathrm{Me}-27), 2.76(1 \mathrm{H}, m, \mathrm{H}-18), 3.15(1 \mathrm{H}, d d, J=10.3$, $4.3 \mathrm{~Hz}, \mathrm{H}-3), 3.60$ (3H, s, MeOOC-28), 5.21 (1H, br s, H-12). ${ }^{13} \mathbf{C}-\mathbf{N M R}\left(\mathbf{C D C l}_{3}\right.$, ppm); 38.4 (C-1), 27.2 (C-2), 79.1 (C-3), 38.8 (C-4), 55.2 (C-5), 18.3 (C-6), 32.8 (C-7), 39.3 (C-8), 47.5 (C-9), 37.1 (C-10), 23.1 (C-11), 122.8 (C-12), 143.6 (C-13), 41.7 (C-14), 28.0 (C-15), 23.4 (C-16), 46.6 (C-17), 41.1 (C-18), 45.8 (C-19), 30.6 (C-20), 33.8 (C-21), 32.1 (C-22), 28.1 (C-23), 15.5 (C-24), 15.4 (C-25), 17.0 (C-26), 25.9 (C-27), 178.6 (C-28), 33.0 (C-29), 23.6 (C-30), 51.5 (MeOOC-28).

Kaempferol-3-O-rutinoside (nicotiflorin, compound 10): UV spectral data (nm); 267, 302sh., $353\left(\mathrm{CH}_{3} \mathrm{OH}\right), 276$, 329, 404 (inc.) $\left(\mathrm{CH}_{3} \mathrm{ONa}\right), 276,306 \mathrm{sh} ., 350,397\left(\mathrm{AlCl}_{3}\right), 277$, 346, $392\left(\mathrm{AlCl}_{3} / \mathrm{HCl}\right), 274,305,370\left(\mathrm{CH}_{3} \mathrm{COONa}\right), 267,354$ $\left(\mathrm{CH}_{3} \mathrm{COONa} / \mathrm{H}_{3} \mathrm{BO}_{3}\right) .{ }^{1} \mathbf{H}-\mathbf{N M R}\left[\left(\mathbf{C D}_{3}\right)_{2} \mathbf{C O}+\mathbf{D}_{2} \mathbf{O}, \mathbf{p p m}\right] ; 6.27$ $(1 \mathrm{H}, d, J=1.6 \mathrm{~Hz}, \mathrm{H}-6), 6.52(1 \mathrm{H}, d, J=1.6 \mathrm{~Hz}, \mathrm{H}-8), 6.97(2 \mathrm{H}$, $\left.d, J=8.8 \mathrm{~Hz}, \mathrm{H}-3^{\prime}, 5^{\prime}\right), 8.12\left(2 \mathrm{H}, d, J=8.8 \mathrm{~Hz}, \mathrm{H}-2^{\prime}, 6^{\prime}\right), 5.14(1 \mathrm{H}$, $\left.d, J=7.2 \mathrm{~Hz}, \mathrm{H}-1^{\prime \prime}\right), 4.56\left(1 \mathrm{H}\right.$, br $\left.s, \mathrm{H}-1^{\prime \prime \prime}\right), 1.09(3 \mathrm{H}, d, J=6.1 \mathrm{~Hz}$, Me-6"'), 3.37-3.63 (Sugar protons).

Quercetin-3-O- $\boldsymbol{\beta}$-D-glucopyranoside (isoquercetin, compound 11): UV spectral data (nm); 258, 270sh., 299sh., $362\left(\mathrm{CH}_{3} \mathrm{OH}\right), 273,330,409$ (inc.) $\left(\mathrm{CH}_{3} \mathrm{ONa}\right), 273,304 \mathrm{sh}$, 370sh., $416\left(\mathrm{AlCl}_{3}\right), 270,299$ sh., 367sh., $363\left(\mathrm{AlCl}_{3} / \mathrm{HCl}\right), 272$, $387\left(\mathrm{CH}_{3} \mathrm{COONa}\right), 262,378\left(\mathrm{CH}_{3} \mathrm{COONa} / \mathrm{H}_{3} \mathrm{BO}_{3}\right) .{ }^{1} \mathbf{H}-\mathbf{N M R}$ $\left[\left(\mathbf{C D}_{3}\right)_{2} \mathbf{C O}+\mathbf{D}_{2} \mathbf{O}, \mathbf{p p m}\right] 6.23(1 \mathrm{H}, b r s, \mathrm{H}-6), 6.47(1 \mathrm{H}, b r s$, H-8), 6.93 (1H, br s, H-5'), $7.56\left(1 \mathrm{H}, d, J=7.9 \mathrm{~Hz}, \mathrm{H}-6^{\prime}\right), 7.80$ $\left(1 \mathrm{H}\right.$, br s, H-2'), $5.23\left(1 \mathrm{H}, d, J=6.4 \mathrm{~Hz}, \mathrm{H}-1^{\prime \prime}\right), 3.25-3.93$ (Sugar protons). ${ }^{13} \mathbf{C}-\mathbf{N M R}\left[\left(\mathbf{C D}_{3}\right)_{2} \mathbf{C O}+\mathbf{D}_{\mathbf{2}} \mathbf{O}, \mathbf{p p m}\right] ; 157.3$ (C-2), 134.4 (C-3), 178.1 (C-4), 161.2 (C-5), 98.9 (C-6), 164.5 (C-7), 94.0 (C8), 157.7 (C-9), $104.3(\mathrm{C}-10), 121.6\left(\mathrm{C}-1^{\prime}\right), 116.8\left(\mathrm{C}-2^{\prime}\right), 144.4$ 
(C-3'), $148.4\left(\mathrm{C}-4^{\prime}\right), 115.3\left(\mathrm{C}-5^{\prime}\right), 121.7\left(\mathrm{C}-6^{\prime}\right), 102.8$ (C-1"), 74.1 (C-2"), 76.3 (C-3"), $69.4\left(\mathrm{C}-4^{\prime \prime}\right), 76.6\left(\mathrm{C}-5^{\prime \prime}\right), 60.9$ (C-6").

Quercetin-3-O- $\beta$-D-galactopyranoside (hyperoside, compound 12): UV spectral data (nm); 258, 270sh., 299sh., $362\left(\mathrm{CH}_{3} \mathrm{OH}\right), 273,330,409$ (inc.) $\left(\mathrm{CH}_{3} \mathrm{ONa}\right), 273,304 \mathrm{sh}$, 370sh., $416\left(\mathrm{AlCl}_{3}\right), 270,299$ sh., 367sh., $363\left(\mathrm{AlCl}_{3} / \mathrm{HCl}\right), 272$, $387\left(\mathrm{CH}_{3} \mathrm{COONa}\right), 262,378\left(\mathrm{CH}_{3} \mathrm{COONa} / \mathrm{H}_{3} \mathrm{BO}_{3}\right) .{ }^{1} \mathbf{H}-\mathbf{N M R}$ [(CD $\left.)_{2} \mathbf{C O}+\mathbf{D}_{2} \mathbf{O}, \mathbf{p p m}\right] ; 6.23(1 \mathrm{H}, b r s, \mathrm{H}-6), 6.47(1 \mathrm{H}, b r s$, $\mathrm{H}-8), 6.93\left(1 \mathrm{H}, b r s, \mathrm{H}-5^{\prime}\right), 7.56\left(1 \mathrm{H}, d, J=7.9 \mathrm{~Hz}, \mathrm{H}-6^{\prime}\right), 7.92$ $\left(1 \mathrm{H}\right.$, br s, H-2'), $5.13\left(1 \mathrm{H}, d, J=7.7 \mathrm{~Hz}, \mathrm{H}-1^{\prime \prime}\right), 3.25-3.93$ (Sugar protons). ${ }^{13} \mathbf{C}-\mathbf{N M R}\left[\left(\mathbf{C D}_{3}\right)_{2} \mathbf{C O}+\mathbf{D}_{2} \mathbf{O}, \mathbf{p p m}\right] ; 157.7$ (C-2), 134.2 (C-3), 178.0 (C-4), 161.2 (C-5), 98.9 (C-6), 164.5 (C-7), 94.0 (C8), 156.9 (C-9), 104.3 (C-10), 121.4 (C-1'), 116.6 (C-2'), 144.4 (C-3'), 148.5 (C-4'), 115.3 (C-5'), $122.0\left(\mathrm{C}-6^{\prime}\right), 103.7$ (C-1") 71.7 (C-2"), 73.4 (C-3"), 68.1 (C-4"), 75.4 (C-5"), 60.1 (C-6").

3, 4-Dihydroxycinnamic acid (caffeic acid, compound 13): UV spectral data (nm); 204sh., 220, 242, 298, $326\left(\mathrm{CH}_{3} \mathrm{OH}\right)$. ${ }^{1} \mathbf{H}-\mathbf{N M R}\left[\left(\mathbf{C D}_{3}\right)_{\mathbf{2}} \mathbf{C O}, \mathbf{p p m}\right] ; 6.22(1 \mathrm{H}, d, J=15.9 \mathrm{~Hz}, \mathrm{H}-8), 6.83$ $(1 \mathrm{H}, d, J=8.2 \mathrm{~Hz}, \mathrm{H}-5), 6.96(1 \mathrm{H}, d d, J=8.1,1.8 \mathrm{~Hz}, \mathrm{H}-6), 7.10$ $(1 \mathrm{H}, d, J=1.8 \mathrm{~Hz}, \mathrm{H}-2), 7.47(1 \mathrm{H}, d, J=15.9 \mathrm{~Hz}, \mathrm{H}-7)$.

\section{Acid hydrolysis of glycosides}

Aliquots (3 mg, each) of the isolated glycosides were separately subjected to acid hydrolysis. The sample was dissolved in $3 \mathrm{ml}$ of $2 \mathrm{~N}$ hydrochloric acid-methanol mixture $(1: 1 \mathrm{v} / \mathrm{v})$ and heated under reflux on a water bath for 2 hours. The reaction mixture was further evaporated under vacuum to dryness, and the residue was suspended in distilled water $(10 \mathrm{ml})$ and then repeatedly extracted with ethyl acetate. The ethyl acetate layer was subjected to TLC (solvent system, $\mathrm{CHCl}_{3}-\mathrm{MeOH}$ 5:1 v/v) alongside reference aglycones. Meanwhile, the aqueous layer was diluted with methanol and evaporated to dryness and the residue obtained was investigated by PC and TLC- (solvent systems: $n$-butanolacetic acid-water 4:1:5 v/v/v, upper layer; and isopropanol:water 7:1 v/v, resp.) for detection of sugar moieties (Mabry et al., 1970). Hydrolysis of compound $\mathbf{1 0}$ afforded D-glucose and L-rhamnose, while the mixture of compounds $\mathbf{1 1}$ and $\mathbf{1 2}$ yielded D-glucose and D-galactose.

Cytotoxic evaluation of the stem bark extract and isolated triterpenoids

Cell lines, culture media, and reference drug

The cell lines were obtained from the American Type Culture Collection (ATCC, Manassas, Virginia, USA) and included human breast adenocarcinoma (MCF-7), human colorectal carcinoma (HCT-116), human hepatocellular carcinoma (HepG2), human osteosarcoma (HOS), and human pulmonary adenocarcinoma (A549) cell lines, alongside telomeraseimmortalized normal human retinal epithelial cell line- (RPE-1).

Cell culture was carried out under sterile conditions using a laminar airflow cabinet biosafety class II level. The culture was maintained in McCoy's 5a medium in case of HCT-116 cell line, Dulbecco's Modified Eagle Medium: Nutrient Mixture F12 in case of A549 and RPE-1 cell lines, and Eagle's Minimum Essential Medium in case of MCF7, HepG2, and HOS cell lines. The culture media were supplied with $1 \%$ antibiotic-antimycotic mixture
$(10,000 \mathrm{U} / \mathrm{ml}$ potassium penicillin, $10,000 \mu \mathrm{g} / \mathrm{ml}$ streptomycin sulfate, and $25 \mu \mathrm{g} / \mathrm{ml}$ amphotericin B), 1\% L-glutamine, and $10 \%$ heat-inactivated fetal bovine serum. Cisplatin was used as a positive control and $0.5 \%$ DMSO solution as a negative control (Thabrew et al., 1997).

\section{Cell viability assay}

The cells were seeded at concentrations of 10,000 cells/ well in case of MCF-7, HepG2, A549, and HOS cell lines and 20,000 cells/well in case of HCT-116 and RPE-1 cell lines, using 96 -well microtiter plastic plates at $37^{\circ} \mathrm{C}$ for 24 hours under $5 \%$ $\mathrm{CO}_{2}$ in a carbon dioxide incubator. Stock solutions of the test isolates were prepared at concentrations of $20,10,5$, and $2.5 \mathrm{mg} /$ $\mathrm{ml}$ for the stem bark methanol extract and $20,10,5$, and $2.5 \mathrm{mM}$ for each isolate. Culture media were aspirated from the cell culture plates, and four different concentrations of the test isolates were prepared, in triplicates. This was carried out by adding an aliquot ( $1 \mu \mathrm{l})$ of each stock solution of the test isolates to fresh medium with cells $(199 \mu \mathrm{l})$ in each well to reach final concentrations of $100,50,25$, and $12.5 \mu \mathrm{g} / \mathrm{ml}$ for the crude extract and 100, 50, 25 , and $12.5 \mu \mathrm{M}$ for each isolate. After incubation for 72 hours, the media were aspirated and $40 \mu \mathrm{l}$ of 3-(4,5-dimethylthiazol-2yl)-2,5-diphenyltetrazolium bromide (MTT) salt $(2.5 \mu \mathrm{g} / \mathrm{ml})$ was added to each well followed by $4 \mathrm{~h}$. incubation at $37^{\circ} \mathrm{C}$ under $5 \%$ $\mathrm{CO}_{2}$. In order to stop the reaction and dissolve the formed crystals, $200 \mu \mathrm{l}$ of $10 \%$ solution of sodium dodecyl sulfate in deionized water was added to each well, followed by incubation overnight, at $37^{\circ} \mathrm{C}$. The absorbance was measured using a microplate multiwell reader at $595 \mathrm{~nm}$. Cell viability was determined using a modified procedure of MTT assay, based on mitochondrial-dependent reduction of the yellow MTT to purple formazan (Mosmann, 1983). The cytotoxicity percentage was calculated as follows:

$$
\% \text { Cytotoxicity }=\left[1-\left(\operatorname{Avg}_{\mathrm{X}} / \operatorname{Avg}_{\mathrm{NC}}\right)\right] \times 100 \text {, }
$$

where Avg indicates average; $\mathrm{X}$ indicates absorbance of sample, and $\mathrm{NC}$ indicates absorbance of the negative control.

The half maximal inhibitory concentration $\left(\mathrm{IC}_{50}\right)$ values of the tested samples were calculated using GraphPad Prism software (version 5.0.), and the selectivity indices (SI) of the cytotoxic samples were deduced from the following equation (Pritchett et al., 2014):

$\mathrm{SI}=\mathrm{IC}_{50}$ of sample against normal cell line $/ \mathrm{IC}_{50}$ of sample against cancer cell line.

The $\mathrm{IC}_{50}$ values and SI of the methanol extract of the stem bark, triterpenoidal isolates, and positive control (cisplatin) against the tested cell lines are recorded in Tables 1 and 2 .

\section{RESULTS AND DISCUSSION}

In an attempt to explore natural compounds with cytotoxic activity, the phytochemical composition of the Egyptian cultivar of $U$. pumila L. was investigated and its cytotoxic potential was assessed. Six compounds, namely, $3 \beta$-acetoxyurs- 11 -en- $13 \beta, 28$ olide, $3 \beta$ - $O$-acetyl ursolic acid, $3 \beta$-O-acetyl oleanolic acid, methyl ursolate, methyl oleanolate, and hyperoside, represented the first reported occurrence in genus Ulmus, whereas the two compounds betulinic acid and nicotiflorin were described from $U$. pumila L. for the first time. Friedelin (Martín-Benito et al., 2011; Wang 
Table 1. $\mathrm{IC}_{50}$ values of the tested samples.

\begin{tabular}{|c|c|c|c|c|c|c|}
\hline \multirow{2}{*}{ Tested samples } & \multicolumn{6}{|c|}{$\mathrm{IC}_{50}$} \\
\hline & MCF-7 & HCT-116 & HepG2 & HOS & A549 & RPE-1* \\
\hline Methanol extract & $29.54 \pm 0.05 \mu \mathrm{g} / \mathrm{ml}$ & $>100 \mu \mathrm{g} / \mathrm{ml}$ & $>100 \mu \mathrm{g} / \mathrm{ml}$ & $>100 \mu \mathrm{g} / \mathrm{ml}$ & $>100 \mu \mathrm{g} / \mathrm{ml}$ & $>100 \mu \mathrm{g} / \mathrm{ml}$ \\
\hline Friedelin & $>100 \mu \mathrm{M}$ & $>100 \mu \mathrm{M}$ & $>100 \mu \mathrm{M}$ & $>100 \mu \mathrm{M}$ & $>100 \mu \mathrm{M}$ & $>100 \mu \mathrm{M}$ \\
\hline $\begin{array}{l}3 \beta \text {-Acetoxyurs-11-en- } \\
13 \beta, 28 \text {-olide }\end{array}$ & $>100 \mu \mathrm{M}$ & $78.98 \pm 0.07 \mu \mathrm{M}$ & $>100 \mu \mathrm{M}$ & $>100 \mu \mathrm{M}$ & $>100 \mu \mathrm{M}$ & $>100 \mu \mathrm{M}$ \\
\hline $\begin{array}{l}3 \beta \text {-O-Acetyl ursolic } \\
\text { acid and/or } 3 \beta \text { - } O \text {-acetyl } \\
\text { oleanolic acid }\end{array}$ & $>100 \mu \mathrm{M}$ & $48.91 \pm 0.12 \mu \mathrm{M}$ & $>100 \mu \mathrm{M}$ & $>100 \mu \mathrm{M}$ & $>100 \mu \mathrm{M}$ & $>100 \mu \mathrm{M}$ \\
\hline Betulinic acid & $22.39 \pm 0.09 \mu \mathrm{M}$ & $22.29 \pm 0.05 \mu \mathrm{M}$ & $>100 \mu \mathrm{M}$ & $>100 \mu \mathrm{M}$ & $42.33 \pm 0.06 \mu \mathrm{M}$ & $31.14 \pm 0.06 \mu \mathrm{M}$ \\
\hline $\begin{array}{l}\text { Methyl ursolate and/or } \\
\text { methyl oleanolate }\end{array}$ & $50.71 \pm 0.18 \mu \mathrm{M}$ & $52.96 \pm 0.07 \mu \mathrm{M}$ & $>100 \mu \mathrm{M}$ & $>100 \mu \mathrm{M}$ & $>100 \mu \mathrm{M}$ & $69.43 \pm 0.2 \mu \mathrm{M}$ \\
\hline
\end{tabular}

*Normal cell line.

$\mathrm{IC}_{50}=$ half maximal inhibitory concentration; MCF-7 = human breast adenocarcinoma; HCT-116 = human colorectal carcinoma; HepG2 = human hepatocellular carcinoma; HOS = human osteosarcoma; A549 = human pulmonary adenocarcinoma; and RPE-1 = telomerase-immortalized normal human retinal epithelial cell line.

Table 2. SI of the cytotoxic samples.

\begin{tabular}{|c|c|c|c|}
\hline \multirow{2}{*}{ Tested samples } & \multicolumn{3}{|c|}{ SI } \\
\hline & MCF-7 & HCT-116 & A549 \\
\hline Methanol extract & $>3.4$ & Inactive & Inactive \\
\hline $3 \beta$-Acetoxyurs-11-en-13 $\beta, 28$-olide & Inactive & $>1.3$ & Inactive \\
\hline $\begin{array}{l}3 \beta \text {-O-Acetyl ursolic acid and/or 3 } 3 \text {-O-acetyl } \\
\text { oleanolic acid }\end{array}$ & Inactive & $>2.0$ & Inactive \\
\hline Betulinic acid & 1.4 & 1.4 & 0.7 \\
\hline Methyl ursolate and/or methyl oleanolate & 1.4 & 1.3 & Inactive \\
\hline
\end{tabular}

MCF-7 = human breast adenocarcinoma; HCT-116 = human colorectal carcinoma; and A549 = human pulmonary adenocarcinoma.

et al., 2006; Zheng et al., 2010), $\beta$-sitosterol, stigmasterol (MartínBenito et al., 2011; Zheng et al., 2010), isoquercetin (Santamour, 1972; Sherman and Giannasi, 1988), and caffeic acid (Zhou et al., 2017) were previously reported from different Ulmus species.

Structure elucidation of the isolates was performed based on spectral analyses (UV, IR, EI-MS, ${ }^{1} \mathrm{H}-$, and ${ }^{13} \mathrm{C}-\mathrm{NMR}$ ) and by comparing the data with literature values. Compounds obtained as pure isolates included friedelin (compound 1) (Mann et al., 2012), 3 $\beta$-acetoxyurs-11-en-13 $\beta, 28$-olide (compound 2) (Raza et al., 2015), betulinic acid (compound 7) (Lee et al., 2005), kaempferol-3-O-rutinoside (nicotiflorin) (compound 10) (Erosa-Rejón et al., 2010), and 3,4-dihydroxycinnamic acid (caffeic acid) (compound 13) (Zhou et al., 2017). In accordance with previous reports (Basir et al., 2014), 3 $\beta$-O-acetyl ursolic acid (compound 3 ) was slightly contaminated with $3 \beta-O$ acetyl oleanolic acid (compound 4). Furthermore, the isolation of $\beta$-sitosterol and stigmasterol (compounds 5 and 6), methyl ursolate, and methyl oleanolate (compounds 8 and 9) as well as quercetin-3-O- $\beta$-D-glucopyranoside (isoquercetin) and quercetin-3- $O-\beta$-D-galactopyranoside (hyperoside) (compounds 11 and 12) as mixtures was in agreement with earlier studies (Furuya et al., 1987; Luhata and Munkombwe, 2015; Pereira et al., 2011).

The cytotoxic efficiency of the stem bark methanol extract (Table 1) might be ascribed to its triterpenoidal components as many of these constituents play an essential role in the upregulation and downregulation of several important genes that influence the apoptotic effects (Prabhu et al., 2011). The isolated betulinic acid was moderately active against MCF-7, HCT-116, and A549 cell lines (respective $\mathrm{IC}_{50}$ values: $22.39 \pm 0.09,22.29 \pm 0.05$, and 42.33 $\pm 0.06 \mu \mathrm{M})$; nevertheless, it was insufficiently selective to MCF-7 and HCT-116 cell lines $(\mathrm{SI}=1.4)$ and lacked selectivity to A549 cell line $(\mathrm{SI}=0.7)$. The cytotoxic potential of betulinic acid was previously explained by its ability to induce apoptosis by directly affecting the mitochondria leading to cleavage of caspase-9 and caspase-3 and activation of nuclear factor-kappa-B (NF-kappa-B), which is a key regulator of stress-induced transcriptional activation (Tripathi et al., 2009). Furthermore, betulinic acid was found to inhibit angiogenesis and metastatic activity through inhibition of aminopeptidase N enzyme (Melzig and Bormann, 1998). Studying the structure-activity relationship of betulinic acid revealed that the skeleton composed of rings $\mathrm{A}, \mathrm{B}$, and $\mathrm{C}$ as well as the carboxylic acid function at $\mathrm{C}-28$ was essential for eliciting its cytotoxic activity (Mukherjee et al., 2006).

\section{CONCLUSION}

The variability in triterpenoidal composition between locally acclimatized $U$. pumila L. samples and those obtained from plants growing abroad could be attributed to environmental conditions. Moreover, the established cytotoxic efficiency of betulinic acid suggests its use as a lead compound for synthesizing potential cytotoxic agents.

\section{AUTHORS' CONTRIBUTIONS}

All authors have contributed to gathering literature data, carrying out the chemical and spectral analyses, interpreting the 
results, and drafting of this manuscript. All authors have read and approved the final manuscript.

\section{ACKNOWLEDGMENTS}

The authors appreciate the sincere efforts of Ms. Therese Labib, Consultant at Orman Botanical Garden and El Qubba Botanical Garden, in identifying and authenticating the plant material.

\section{CONFLICT OF INTEREST}

The authors declare that there are no competing interests

\section{FUNDING} Giza, Egypt.

The work was funded by the National Research Centre,

\section{REFERENCES}

Basir D, Julinar AE, Untari B. Oxidation and acetylation of ursolic and oleanolic acids isolated from Fagraea fragrans fruits: antiproliferation of P388 leukemia cells. Indones J Chem, 2014; 14(3):269-76.

Bora KS, Kumar A, Bisht G. Evaluation of antimicrobial potential of successive extracts of Ulmus wallichiana Planch. J Ayurveda Integr Med, 2017; XXX:1-5.

Boudaoud-Ouahmed HY, Ouaret N, Schini-Keirth VB, Djebbli $\mathrm{N}$, Atmani D. Evaluation of gastroprotective, hepatoprotective and hypotensive activities of Ulmus campestris bark extract. Phytothérapie, $2015 ; 14: 229-40$

Burden RS, Kemp MS. Sesquiterpene phytoalexins from Ulmus glabra. Phytochemistry, 1984; 23(2):383-5.

Duke JA, Ayensu ES. Medicinal plants of China. Reference Publications Inc, Algonac, MI, 1985.

Encyclopaedia Britannica. 2019. Available via https://www. britannica.com/plant/Ulmaceae (Accessed 20 September 2019).

Erosa-Rejón GM, Peña-Rodríguez L, Sterner V. Isolation of kaempferol-3-rutinoside from the leaf extract of Sideroxylon foetidissimum subsp. Gaumeri. Rev Latinoam Quim, 2010; 38(1):7-11.

Furuya T, Orihara Y, Hayashi C. Triterpenoids from Eucalyptus perriniana cultured cells. Phytochemistry, 1987; 26(3):715-9.

Ghosh C, Chung HY, Nandre RM, Lee JH, Jeon TI, Kim IS, Yang SH, Hwang SG. An active extract of Ulmus pumila inhibits adipogenesis through regulation of cell cycle progression in 3T3-L1 cells. Food Chem Toxicol, 2012; 50(6):2009-15

Hamed MM, El-Amin S, Refahy L, Soliman ESA, Mansour WA, Abu Taleb HM, Morsi E. Anticancer and antiviral estimation of three Ulmus pravifolia extracts and their chemical constituents. Orient J Chem, 2015; 31(3):1621-34

Joo T, Sowndhararajan K, Hong S, Lee J, Park SY, Kim S, Jhoo JW. Inhibition of nitric oxide production in LPS-stimulated RAW 264.7 cells by stem bark of Ulmus pumila L. Saudi J Biol Sci, 2014; 21(5):427-35.

Jung HJ, Jeon HJ, Lim EJ, Ahn EK, Song YS, Lee S, Shin KH, Lim CJ, Park EH. Anti-angiogenic activity of the methanol extract and its fractions of Ulmus davidiana var. japonica. J Ethnopharmacol, 2007; 112(2):406-9.

Kim SI, Sim KH, Choi HY. A comparative study of antioxidant activity in some Korean medicinal plants used as food materials. Mol Cell Toxicol, 2010; 6(3):279-85.

Lee JH, Lee YK, Choi YR, Park J, Jung SK, Chang YH. The characterization, selenylation and anti-inflammatory activity of pectic polysaccharides extracted from Ulmus pumila L. Int J Biol Macromol, 2018; 111:311-8

Lee TH, Chiou JL, Lee CK, Kuo YH. Separation and determination of chemical constituents in the roots of Rhus Javanica L. var. Roxburghian. J Chin Chem Soc, 2005; 52:833-41.
Luhata PL, Munkombwe MN. Isolation and characterisation of stigmasterol and $\beta$-sitosterol from Odontonema strictum (Acanthaceae). J Innov Pharm Biol Sci, 2015; 2(1):88-95.

Mabry TJ, Markham KR, Thomas MB. The systematic identification of flavonoids. Springer-Verlag, New York, NY, 1970.

Mann A, Ibrahim K, Oyewale AO, Amupitan J, Fatope M, Okogun J. Antimycobacterial friedelane-terpenoid from the root bark of Terminalia avicennioides. Am J Chem, 2012; 1(2):52-5.

Martín-Benito D, García-Vallejo M, Pajares J, López D Triterpenes in elms in Spain. Can J For Res, 2011; 35:199-205.

Melzig MF, Bormann H. Betulinic acid inhibits aminopeptidase N activity. Planta Med, 1998; 64:655-7.

Mina SA, Melek FR, Adeeb RM, Hagag EG. LC/ESI-MS/ MS profiling of Ulmus parvifolia extracts and evaluation of its antiinflammatory, cytotoxic, and antioxidant activities. Z Naturforsch C Biosci, 2016; 71(11-12):415-21.

Mosmann T. Rapid colorimetric assay for cellular growth and survival: application to proliferation and cytotoxicity assays. J Immunol Methods, 1983; 65(1):55-63.

Mukherjee R, Kumar V, Srivastava SK, Agarwal SK, Burman AC. Betulinic acid derivatives as anticancer agents: structure activity relationship. Anticancer Agents Med Chem, 2006; 6:271-9.

Pereira C, Barreto C, Kuster RM, Simas N, Sakuragui C, Porzel A, Wessjohann L. Flavonoids and a neolignan glucoside from Guarea macrophylla (Meliaceae). Quím Nova, 2011; 35(6):1123-6.

Prabhu A, Krishnamoorthy M, Prasad DJ, Naik P. Anticancer activity of friedelin isolated from ethanolic leaf extract of Cassia tora on HeLa and HSC-1 cell lines. Indian J Appl Res, 2011; 3(10):1-4.

Pritchett JC, Naesens L, Montoya J. Chapter 19- treating HHV-6 infections: the laboratory efficacy and clinical use of anti-HHV-6 agents. In: Flamand L, Lautenschlager I, Krueger GRF, Ablashi DV, Dhraram V (ed.). Human herpesviruses HHV-6A, HHV-6B and HHV-7. Diagnosis and clinical management. 3rd edition. Elsevier, Amsterdam, Netherlands, pp 311-31, 2014

Raza R, Ilyas Z, Ali S, Nisar M, Khokhar MY, Iqbal J. Identification of highly potent and selective $\alpha$-glucosidase inhibitors with antiglycation potential, isolated from Rhododendron arboretum. Rec Nat Prod, 2015; 9(2):262-6.

Richens RH. Elm. Cambridge University Press, Cambridge, UK, 1983.

Santamour FS. Flavonoid distribution in Ulmus. Bull Torrey Bot Club, 1972; 99(3):127-31.

Sherman SL, Giannasi DE. Foliar flavonoids of Ulmus in Eastern North America. Biochem Syst Ecol, 1988; 16(1):51-6.

So HM, Yu JS, Khan Z, Subedi L, Ko YJ, Lee IK, Park WS, Chung SJ, Ahn MJ, Kim SY, Kim KH. Chemical constituents of the root bark of Ulmus davidiana var. japonica and their potential biological activities. Bioorg Chem, 2019; 91:103145.

Thabrew MI, Hughes RD, McFarlane IG. Screening of hepatoprotective plant components using a HepG2 cell cytotoxicity assay. J Pharm Pharmacol, 1997; 49(11):1132-5.

Tripathi L, Kumar P, Singh R. A review on extraction, synthesis and anticancer activity of betulinic acid. Curr Bioact Compd, 2009; 5: $160-8$

Wang D, Xia M, Cui Z. New triterpenoids isolated from the roo bark of Ulmus pumila L. Chem Pharm Bull, 2006; 54(6):775-8.

Wang D, Xia M, Cui Z, Tashiro S, Onodera S, Ikejima T. Cytotoxic effects of mansonone $\mathrm{E}$ and $\mathrm{F}$ isolated from Ulmus pumila. Biol Pharm Bull, 2004; 27(7):1025-30.

Watson L, Dallwitz MJ. The Families of Flowering Plants: Ulmaceae Mirb. 1992. Available via https://www.delta-intkey.com/angio/ www/ulmaceae.html (Accessed 10 October 2019).

You YO, Choi NY, Kim KJ. Ethanol extract of Ulmus pumila root bark inhibits clinically isolated antibiotic-resistant bacteria. Evid Based Complementary Altern Med, 2013; 2013:269874. 
Zheng M, Yang JH, Li Y, Li X, Chang H, Son J. Antiinflammatory activity of constituents isolated from Ulmus davidiana var. japonica. Biomol Ther, 2010; 18(3):321-8.

Zhou Z, Shao H, Han X, Wang K, Gong C, Yang X. The extraction efficiency enhancement of polyphenols from Ulmus pumila $\mathrm{L}$. barks by trienzyme-assisted extraction. Ind Crops Prod, 2017; 97:401-8.
How to cite this article:

Melek FR, Zalabani SME, Ghaly NS, Sabry OM, Fayad W, Boulis AG. Phytoconstituents with cytotoxic activity from Ulmus pumila L. J Appl Pharm Sci, 2021; 11(05):127-134. 